\title{
RIGIDITY OF PSEUDO-HOLOMORPHIC CURVES OF CONSTANT CURVATURE IN GRASSMANN MANIFOLDS
}

\author{
QUO-SHIN CHI AND YUNBO ZHENG
}

\begin{abstract}
Rigidity of minimal immersions of constant curvature in harmonic sequences generated by holomorphic curves in Grassmann manifolds is studied in this paper by lifting them to holomorphic curves in certain projective spaces. We prove that for such curves the curvature must be positive, and that all such simply connected curves in $C P^{n}$ are generated by Veronese curves, thus generalizing Calabi's counterpart for holomorphic curves in $C P^{n}$. We also classify all holomorphic curves from the Riemann sphere into $G(2,4)$ whose curvature is equal to 2 into two families, which illustrates pseudo-holomorphic curves of positive constant curvature in $G(m, N)$ are in general not unitarily equivalent, constracting to the fact that generic isometric complex submanifolds in a Kaehler manifold are congruent.
\end{abstract}

\section{INTRODUCTION}

Given a harmonic map $f$ from a Riemann surface $M$ into the complex Grassmann manifold $G(m, N)$, by using the $\partial$-transform associated to the map $f$, S. S. Chern and J. G. Wolfson obtained the following sequence of harmonic maps [6],

$$
f=f_{0} \rightarrow f_{1} \rightarrow f_{2} \rightarrow \ldots
$$

where $f_{j+1} \equiv \partial f_{j}$ for $j=0,1,2, \ldots$, and $f_{j}: M \rightarrow G\left(m_{j}, N\right)$ are harmonic maps; one defines $m_{j+1}=0$ if $f_{j}$ is anti-holomorphic. We call $f_{r}$ a pseudoholomorphic curve of position $r$ generated by $f$ when $f$ is holomorphic, where $f$ is called the directrix of $f_{r}$. Such curves with the induced metrics from the associated Grassmannians form a class of minimal immersions. When specialized to $G(1, n+1)=C P^{n}$, a pseudo-holomorphic curve of position $r$ is just the $r$ th position vector of a Frenet frame of a holomorphic curve projected into $C P^{n}$. The importance of such maps comes from the fact that any harmonic map from the Riemann sphere, or any harmonic map of nonzero degree from a torus, into $C P^{n}$ is pseudo-holomorphic [7]. Furthermore in [6] these curves play a central role, when $m_{j}$ 's defined in the sequence $(0.1)$ are all equal and $f_{j}$ 's are orthogonal, called Frenet harmonic sequences, for the study of

Received by the editors April 27, 1987 and, in revised form, January 11, 1988. This paper was presented at the AMS Nebraska meeting, October 31, 1987, by the second author.

1980 Mathematics Subject Classification (1985 Revision). Primary 53C42, 53C55.

Key words and phrases. Pseudo-holomorphic curves, Grassmann manifolds, Veronese curves. 
harmonic maps from the Riemann sphere into $G(2, n)$. Henceforth they have attracted much attention.

When a simply connected pseudo-holomorphic curve is of position zero in $C P^{n}$, i.e., when it is holomorphic, Calabi showed that the only such curves of constant curvature are obtained, up to unitary equivalence, by the Veronese curves restricted to suitable domains of the Riemann sphere in a linear subspace of $C P^{n}$; furthermore any two isometric holomorphic curves in $C P^{n}$ must be unitarily congruent $[3,4]$. In this paper we generalize Calabi's results in the following way. On the one hand we show that there is no (local) pseudoholomorphic curve of nonpositive constant curvature in $G(m, N)$ (Theorem 1), and that all simply connected pseudo-holomorphic curves of positive constant curvature in $C P^{n}$ are unitarily equivalent to the ones generated by the Veronese curves restricted to suitable domains in linear subspaces of $C P^{n}$ (Theorem 2). Notice that the two theorems are completely local in nature, where no topological assumptions are needed. Theorem 2 implies previous results obtained in [1] that any pseudo-holomorphic curve of constant curvature generated by a rational normal curve in $C P^{n}$ is unitarily equivalent to one generated by some Veronese curve; and also obtained in Bando and Ohnita, Minimal 2-spheres with constant curvature in $P_{n}(\mathbf{C})$, (J. Math. Soc. Japan 39 (1987), sent to us by the authors after this present paper was done), that any minimal immersion of the Riemann sphere into $C P^{n}$ of constant curvature is unitarily equivalent to one generated by some Veronese curve. We would like to point out that the Riemann surfaces being spheres is crucial in Bando and Ohnita's analysis in that they need the facts that the minimal immersions are thus pseudo-holomorphic and that there are no nontrivial harmonic functions on a Riemann sphere.

On the other hand M. Green showed that generic isometric complex submanifolds in a Kaehler manifold are congruent [9]. We shall classify in Theorem 3 the holomorphic curves from the Riemann sphere into $G(2,4)$ with the induced constant curvature 2 into two classes, up to unitary equivalence, in which none of the curves are congruent. This shows that the holomorphic curves of constant curvature in the Grassmannians are among the "nongeneric" ones, making the classification of such curves pretty interesting.

The unitary equivalence fails to be true in general for any two isometric pseudo-holomorphic curves in $C P^{n}$. For example, the complex conjugate of any holomorphic curve is pseudo-holomorphic and is isometric, but not unitarily equivalent, to the holomorphic curve. Nevertheless one can still ask whether they are generated by congruent holomorphic curves when the two pseudoholomorphic curves are of the same position (In [1] it is proved true in case when the two isometric curves have the same Kaehler angles.) More generally it would be interesting to understand the space of noncongruent pseudoholomorphic curves in $C P^{n}$ which are isometric to a given one. ${ }^{1}$

\footnotetext{
${ }^{1}$ The first author has shown that a large class of pseudo-holomorphic curves from a compact Riemann surface into $C P^{2}$ are rigid up to unitary equivalence and the complex conjugation.
} 
Our starting point is the lifting holomorphic map defined in (1.17), which transforms the metric of a pseudo-holomorphic curve into that of a holomorphic curve. By studying this lifting map, one can conclude the above theorems via the corresponding counterparts of Calabi on holomorphic curves in $C P^{n}$. Another consequence of this construction is that the holomorphic liftings of two isometric pseudo-holomorphic curves must be unitarily congruent, which seems to be a first step towards understanding the rigidity of such curves (Proposition 1).

Basic material is developed in $\S 1$, where we collect formulas relating certain invariants of pseudo-holomorphic curves by using the method of moving frames. These invariants have been studied before for various purposes $[1,15]$. Construction of the lifting map (1.17) enables us to investigate these invariants, which leads to the proof of Theorem 1 in $\S 2$ and Theorem 2 in $\S 3$. The proof of Theorem 3 in $\S 4$ is based on some previous work done by G. Jensen [12].

We would like to thank Professor Gary Jensen, the second author's advisor, for extensive discussion and kindly showing us their results in [1], which initiated this study.

\section{PSEUdo-hOlOMORPHIC CURVES AND THE LIFTING MAPS}

The complex Grassmann manifold $G(m, N)$ is the set of all $m$-dimensional linear subspaces of $C^{N}$ through the origin, which can be realized as the homogeneous space $U(N) /(U(m) \times U(N-m))$, where

$$
\begin{aligned}
\pi: U(N) & \rightarrow G(m, N), \\
g & \rightarrow[g \cdot o]
\end{aligned}
$$

is a principal $U(m) \times U(N-m)$-bundle, $O$ is the $N \times m$ matrix

$$
\left[\begin{array}{c}
I_{m} \\
0
\end{array}\right]
$$

and $I_{m}$ is the $m \times m$ identity matrix.

Let $W_{A B}$ be the Maurer-Cartan forms of $U(N), 1 \leq A, B \leq N$, and let $e$ be a local section of $\pi: U(N) \rightarrow G(m, N)$. Set

$$
\begin{aligned}
\Psi_{A B} & =e^{*}\left(W_{A B}\right), \\
d s_{G(m, N)}^{2} & =\sum_{\substack{1 \leq i \leq m \\
m<\alpha \leq N}} \Psi_{i \alpha} \bar{\Psi}_{i \alpha} .
\end{aligned}
$$

Then $d s_{G(m, N)}^{2}$ is a $U(N)$-invariant hermitian metric on $G(m, N)$; when $m=$ 1 and $N=n+1$ this is the Fubini-Study metric on $C P^{n}$ with constant holomorphic curvature 4.

Suppose now $M$ is a Riemann surface, and $f: M \rightarrow G(m, N)$ is a smooth immersion inducing the metric $d s_{M}^{2}$. Locally

$$
\begin{gathered}
d s_{M}^{2}=\theta \bar{\theta}, \\
d \theta_{M}^{2}=i \omega \wedge \theta,
\end{gathered}
$$


where $\theta$ is a local unitary coframe on $M$ and $\omega$ is the real-valued connection form associated to the metric $d s_{M}^{2}$. Also

$$
d \omega=\frac{i}{2} K \theta \wedge \bar{\theta},
$$

where $K$ is the Gaussian curvature of $d s_{M}^{2}$.

Now assume that $f_{r}$ is a pseudo-holomorphic map from $M$ to $G(m, N)$ generated by the directrix $f$. We first note that the pseudo-holomorphic sequence $(0.1)$ generated by $f$ must terminate after finite steps. To see this it suffices to show that the $f_{i}$ 's in the sequence are mutually orthogonal. However this follows by an easy induction argument from the fact that $\bar{\partial} \partial\left(f_{i}\right) \subset f_{i},[16]$, and that $f_{0}=f$ is holomorphic. For notational simplicity we may assume that $f$ is linearly full so that $N=\sum_{j=0}^{k} m_{j}$. Choose a suitable local unitary frame $e$ along $f$, where

$$
e=\left(e_{01}, \ldots, e_{0 m_{0}}, e_{11}, \ldots, e_{1 m_{1}}, \ldots, e_{k m_{k}}\right)
$$

and $e_{j 1}, \ldots, e_{j m_{j}}$ span $f_{j}$ for $0 \leq j \leq k$, and let $\phi=e^{*}(W)$. Then

$$
\phi=\left[\begin{array}{cccccc}
\varphi_{0} & -\bar{X}_{01} \bar{\theta} & 0 & & & \\
X_{01} \theta & \varphi_{1} & -\bar{X}_{12} \bar{\theta} & & & 0 \\
0 & X_{12} \theta & \varphi_{2} & & & \\
& & & \ddots & & \\
& 0 & & & \varphi_{k-1}, & -\bar{X}_{k-1, k} \bar{\theta} \\
X_{k-1, k} \theta & \varphi_{k}
\end{array}\right]
$$

where $\varphi_{j}$ are $u\left(m_{j}\right)$-valued 1-forms,

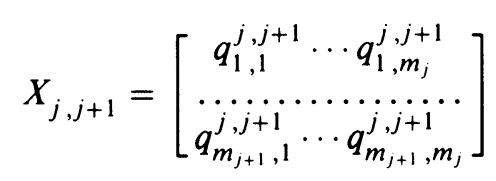

are complex $m_{j+1} \times m_{j}$-matrix-valued smooth functions for $0 \leq j \leq k-1$ and $\theta$ is a local unitary coframe on $M$ with respect to the induced metric $f_{r}^{*} d s_{G(m, N)}^{2}$.

For each $0 \leq j \leq k-1$, define

$$
\left|X_{j, j+1}\right|^{2}=\operatorname{trace}\left(X_{j, j+1} \cdot{ }^{t} \bar{X}_{j, j+1}\right) \text {. }
$$

Since the metric may have isolated singularities, $\left|X_{j, j+1}\right|^{2}$ is well defined on a connected open dense subset $U$ of $M$ and there

$$
\left|X_{r-1, r}\right|^{2}+\left|X_{r, r+1}\right|^{2}=1 \text {. }
$$

Here we set $\left|X_{-1,0}\right|=\left|X_{k, k+1}\right|=0$.

Notice that when $m_{j}=m$ for $j=0,1, \ldots, k-1,\left|\operatorname{det}\left(X_{j, j+1}\right)\right|$ are global invariants of analytic type defined on $U$ vanishing only at isolated points, and 
around the points where $\operatorname{det}\left(X_{j, j+1}\right) \neq 0$ they satisfy [15]

$$
\begin{aligned}
& \Delta \log \left|\operatorname{det}\left(X_{j, j+1}\right)\right| \\
& \quad=m K+2\left(\left|X_{j-1, j}\right|^{2}+\left|X_{j+1, j+2}\right|^{2}-2\left|X_{j, j+1}\right|^{2}\right),
\end{aligned}
$$

for $0 \leq j \leq k-1$. In fact it is shown in [16] that when $m_{j}$ 's are different one can still associated with $X_{j, j+1}$ a global invariant of analytic type which reduces to $\operatorname{det}\left(X_{j, j+1}\right)$ when $m_{j}$ 's are equal. But we will not use this fact later on.

Notice also that when $m=1$, if we set

$$
q_{j}=\left|X_{j, j+1}\right|=\left|\operatorname{det}\left(X_{j, j+1}\right)\right|,
$$

then $(1.10)$ reduces to

$$
q_{r-1}^{2}+q_{r}^{2}=1
$$

and (1.11) reduces to

$$
\Delta \log q_{j}=K+2\left(q_{j-1}^{2}+q_{j+1}^{2}-2 q_{j}^{2}\right) .
$$

Now let $P: G(m, N) \rightarrow C P^{\left(\begin{array}{c}N \\ m\end{array}\right)-1}$ be the standard Plücker embedding [11]. Then for each $i, P\left(f_{i}(p)\right)$ is an $\left(\begin{array}{l}N \\ m_{i}\end{array}\right)$-dimensional vector defined up to a multiple. Also, for $0 \leq j \leq k$ set

$$
F_{j}=P\left(f_{0}\right) \wedge P\left(f_{1}\right) \wedge \cdots \wedge P\left(f_{j}\right) .
$$

$\left[F_{j}\right]$ is a holomorphic map from $M$ to $C P^{L(j)-1}$, where

$$
L(j)=\left(\begin{array}{c}
N \\
\sum_{i=0}^{j^{N}} m_{i}
\end{array}\right),
$$

called the $j$ th associated curve of $f$; when $m=1$, this is just the standard $j$ th associated curve of the holomorphic curve $f$ in $C P^{N-1}$ and the pull-back metric by $F_{j}$ can be expressed as [1]

$$
F_{j}^{*} d s_{C P_{j+1}^{(N)}}^{2}=q_{j}^{2} \theta \bar{\theta}
$$

Finally we define $\eta_{j-1, j}: M \rightarrow C P^{N(j)}$, where

$$
N(j)=L(j-1) L(j)-1,
$$

for $1 \leq j \leq k$ by letting

$$
\eta_{j-1, j}(p)=\left[F_{j-1}(p) \otimes F_{j}(p)\right] .
$$

It is easy to check that this is a well-defined holomorphic curve in $C P^{N(j)}$. We will call it the $j$ th lifting map of $f$, which will play an important role in our analysis in the sequel. 
2. NONEXISTENCE OF PSEUDO-HOLOMORPHIC CURVES IN $G(m, N)$ WITH NONPOSITIVE CONSTANT CURVATURE

Without loss of generality one may assume that the metrics induced by pseudo-holomorphic curves are nonsingular on $M$, since the following argument is local.

A nonconstant holomorphic curve in a complex projective space is a pseudoholomorphic curve of position zero. For such a curve one has $\left|X_{0,1}\right|^{2}=1$ by (1.10). Suppose now that the holomorphic curve has constant curvature. Then by (1.10) all $\left|X_{j, j+1}\right|$ are constant, and hence $K=4 / N_{0}$ for some positive integer.

Now assume that $f_{r}$ is a pseudo-holomorphic curve of position $r$ with constant Gaussian curvature $K$ generated by a holomorphic curve $f$ in $G(m, N)$.

If $r=0, f_{0}=f$ is holomorphic. Via the standard Plücker embedding $P$, $P \circ f$ is a holomorphic curve in $C P^{\left(\begin{array}{c}N \\ m\end{array}\right)-1}$ with the same constant curvature $K$. Therefore $K=4 / N_{0}>0$ by the above remark.

If $r>0$, let $\eta_{r-1, r}$ be the $r$ th lifting map defined by (1.17) and let $e$ be the local orthonormal frame defined by (1.6). Then locally

$$
\eta_{r-1, r}=\left[\left(e_{01} \wedge \cdots \wedge e_{(r-1), m_{r-1}}\right) \otimes\left(e_{01} \wedge \cdots \wedge e_{r m_{r}}\right)\right] .
$$

By (1.7), (1.8), (1.9), (1.10) and Maurer-Cartan structure equations, one gets

$$
\begin{aligned}
d\left\{\left(e_{01} \wedge \cdots \wedge e_{(r-1), m_{r-1}}\right) \otimes\left(e_{01} \wedge \cdots \wedge e_{r m_{r}}\right)\right\} \\
=\left(\sum_{i=0}^{r-1} \operatorname{trace} \varphi_{i}+\sum_{i=0}^{r} \operatorname{trace} \varphi_{i}\right)\left\{\left(e_{01} \wedge \cdots \wedge e_{(r-1), m_{r-1}}\right) \otimes\left(e_{01} \wedge \cdots \wedge e_{r m_{r}}\right)\right\} \\
+\sum_{i=1}^{m_{r}} \sum_{t=1}^{m_{r-1}} q_{i, t}^{r-1, r} \theta\left\{\left(e_{01} \wedge \cdots \wedge e_{(r-1),(i-1)} \wedge e_{r i}\right.\right. \\
\left.\left.\wedge e_{(r-1),(i+1)} \wedge \cdots \wedge e_{(r-1), m_{r-1}}\right) \otimes\left(e_{01} \wedge \cdots \wedge e_{r m_{r}}\right)\right\} \\
+\sum_{i=1}^{m_{r+1}} \sum_{t=1}^{m_{r}} q_{i, t}^{r, r+1} \theta\left\{\left(e_{01} \wedge \cdots \wedge e_{(r-1), m_{r-1}}\right)\right. \\
\left.\otimes\left(e_{01} \wedge \cdots \wedge e_{r,(i-1)} \wedge e_{(r+1), i} \wedge e_{r,(i+1)} \wedge \cdots \wedge e_{r m_{r}}\right)\right\}
\end{aligned}
$$

and hence

$$
\begin{aligned}
\eta_{r-1, r}^{*} d s_{C P^{N(r)}}^{2} & =\left(\sum_{i=1}^{m_{r}} \sum_{t=1}^{m_{r-1}}\left|q_{i, t}^{r-1, r}\right|^{2}+\sum_{i=1}^{m_{r+1}} \sum_{t=1}^{m_{r}}\left|q_{i, t}^{r, r+1}\right|^{2}\right) \theta \bar{\theta} \\
& =\left(\left|X_{r-1, r}\right|^{2}+\left|X_{r, r+1}\right|^{2}\right) \theta \bar{\theta}=\theta \bar{\theta}=f_{r}^{*} d s_{G(m, N)}^{2} .
\end{aligned}
$$

Therefore the pull-back metrics induced by $f_{r}$ and by the lifting map $\eta_{r-1, r}$ are identical, and in particular, their Gaussian curvatures are equal. Since $\eta_{r-1, r}$ is holomorphic, one infers $K=4 / N_{0}$ for some positive integer $N_{0}$. Thus one can conclude the following. 
Theorem 1. There does not exist any nonconstant (local) pseudo-holomorphic curve of nonpositive constant Gaussian curvature in $G(m, N)$.

Corollary 1. All minimal tori of constant curvature in $S^{n}$ are not pseudo-holomorphic, when they are regarded as curves in $C P^{n}$ via the standard embedding of $S^{n}$ into $C P^{n}$.

Minimal tori of constant curvature in $S^{n}$ have been classified in [2, 13]. These surfaces are of degree zero when imbedded in $C P^{n}$, which provide a large class of examples verifying that Eells and Woods' nonzero degree condition mentioned in the introduction cannot be dropped. One also establishes the following proposition.

Proposition 1. The holomorphic liftings of two isometric pseudo-holomorphic curves in $G(m, N)$ must be unitarily equivalent.

Proof. Use the fact that two isometric holomorphic curves in a complex projective space are unitarily congruent.

\section{RIGIDITY OF PSEUDO-HOLOMORPHIC CURVES OF POSITIVE CONSTANT CURVATURE IN $C P^{n}$}

Restricting to pseudo-holomorphic curves of positive constant curvature in $C P^{n}$, where now $N=n+1, m=1$, and $N(j)=\left(\begin{array}{c}N \\ j\end{array}\right)\left(\begin{array}{c}N \\ j+1\end{array}\right)-1$, classical examples are the Veronese curves $V_{m}: C P^{1} \rightarrow C P^{m} \subset C P^{n}$ given by

$$
V_{m}[(z, w)]=\left[\left(\left(\begin{array}{c}
m \\
0
\end{array}\right)^{1 / 2} w^{m},\left(\begin{array}{c}
m \\
1
\end{array}\right)^{1 / 2} z w^{m-1}, \ldots,\left(\begin{array}{c}
m \\
m
\end{array}\right)^{1 / 2} z^{m}\right)\right]
$$

whose curvatures are $4 / m$ for $0 \leq m \leq n$. The following theorem says that they generate all pseudo-holomorphic curves of constant curvature in $C P^{n}$.

Theorem 2. Let $M$ be a simply connected Riemann surface not necessarily closed, and let $f: M \rightarrow C P^{n}$ be a pseudo-holomorphic curve of constant curvature. Then the curve is unitarily equivalent to an open subset of a pseudoholomorphic curve generated by some Veronese curve in a linear subspace of $C P^{n}$ after reparameterization.

Proof. Notation as in $\S 1$. Let $f_{r}$ be a pseudo-holomorphic curve in $C P^{n}$ of position $r$ with constant curvature $K$. Without loss of generality, we may assume that $f_{r}$ is linearly full. As has been shown in $\S 2, K=4 / N_{0}$ for some positive integer $N_{0}$, which is also the curvature for the metric $\eta_{r-1, r}^{*} d s_{C P^{N(r)}}^{2}$. Since $\eta_{r-1, r}$ is holomorphic, by the theorem of Calabi $\eta_{r-1, r}$ is unitarily equivalent to a portion of $V_{N_{0}}$, a Veronese curve in a linear subspace of $C P^{N(r)}$ after reparameterization.

Let $\widetilde{F}_{r}=\left(F_{r}^{0}, F_{r}^{1}, \ldots, F_{r}^{\left(\begin{array}{c}N \\ r+1\end{array}\right)-1}\right)$ and $\widetilde{F}_{r-1}=\left(F_{r-1}^{0}, F_{r-1}^{1}, \ldots, F_{r-1}^{\left(\begin{array}{c}N \\ r\end{array}\right)-1}\right)$ be local lifts of $F_{r}$ and $F_{r-1}$ respectively, where $F_{r}^{t}$ and $F_{r-1}^{i}$ are local holomorphic 
functions for $0 \leq t \leq\left(\begin{array}{c}N \\ r+1\end{array}\right)-1$, and $0 \leq i \leq\left(\begin{array}{l}N \\ r\end{array}\right)-1$. Then

$$
\begin{aligned}
\tilde{\eta}_{r-1, r} & =\widetilde{F}_{r} \otimes \widetilde{F}_{r-1} \\
& =\left(F_{r}^{0} F_{r-1}^{0}, F_{r}^{0} F_{r-1}^{1}, \ldots, F_{r}^{i} F_{r-1}^{j}, \ldots, F_{r}^{\left(\begin{array}{c}
N \\
r+1
\end{array}\right)-1} F_{r-1}^{\left(\begin{array}{c}
N \\
r
\end{array}\right)-1}\right)
\end{aligned}
$$

is a local lift of $\eta_{r-1, r}$. Since $\eta_{r-1, r}$ and $V_{N_{0}}$ are unitarily equivalent,

$$
\tilde{\eta}_{r-1, r}=A \cdot \widetilde{V}_{N_{0}} \cdot h \text {, }
$$

where $h$ is a nowhere vanishing local holomorphic function, $A$ is a suitable constant unitary matrix, and $\widetilde{V}_{N_{0}}$ is the standard lift of $V_{N_{0}}$ in $C^{N_{0}+1} \subset C^{N(r)+1}$.

Comparing components of both sides of (3.2) one sees

$$
F_{r}^{i} F_{r-1}^{j}(z)=P^{i j}(z) h(z),
$$

where $P^{i j}$ are polynomials in $z$ for $0 \leq i \leq\left(\begin{array}{c}N \\ r+1\end{array}\right)-1$ and $0 \leq j \leq\left(\begin{array}{c}N \\ r\end{array}\right)-1$.

Without loss of generality, one may assume that $F_{r}^{0} F_{r-1}^{0} \neq 0$. Then

$$
\frac{F_{r}^{i}}{F_{r}^{0}}=\frac{F_{r}^{i} F_{r-1}^{0}}{F_{r}^{0} F_{r-1}^{0}}=\frac{P^{i, 0} h}{P^{0,0} h}=\frac{P^{i, 0}}{P^{0,0}},
$$

and

$$
\frac{F_{r-1}^{j}}{F_{r-1}^{0}}=\frac{F_{r}^{0} F_{r-1}^{j}}{F_{r}^{0} F_{r-1}^{0}}=\frac{P^{0, j} h}{P^{0,0} h}
$$

so

$$
F_{r-1}=\left[\left(h \cdot P^{0,0}, h \cdot P^{0,1}, \ldots, h \cdot P^{0,\left(\begin{array}{c}
N \\
r
\end{array}\right)-1}\right)\right]
$$

and

$$
F_{r}=\left[\left(P^{0,0}, P^{1,0}, \ldots, P^{\left(\begin{array}{c}
N \\
r+1
\end{array}\right)-1,0}\right)\right] .
$$

Thus one may assume further that

$$
\widetilde{F}_{r-1}=\left(h P^{0,0}, h P^{0,1}, \ldots, h P^{0,\left(\begin{array}{c}
N \\
r
\end{array}\right)-1}\right)
$$

and

$$
\widetilde{F}_{r}=\left(P^{0,0}, P^{1,0}, \ldots, P^{\left(\begin{array}{c}
N \\
r+1
\end{array}\right)-1,0}\right) .
$$

For $v=\left(v_{0}, v_{1}, \ldots, v_{L}\right)$ in $C^{L+1}$, define $\|v\|^{2}=\sum_{i=0}^{L}\left|v_{i}\right|^{2}$. Then by (3.2) one gets

$$
\left\|\tilde{\eta}_{r-1, r}\right\|^{2}=\left\|A \cdot \widetilde{V}_{N_{0}}\right\|^{2}|h|^{2}=\left\|\widetilde{V}_{N_{0}}\right\|^{2}|h|^{2}=\left(1+|z|^{2}\right)^{N_{0}}|h|^{2} .
$$

On the other hand,

$$
\left\|\tilde{\eta}_{r-1, r}\right\|^{2}=|h|^{2}\left(\sum_{j=0}^{\left(\begin{array}{c}
N \\
r
\end{array}\right)-1}\left|P^{0, j}\right|^{2}\right)\left(\sum_{j=0}^{\left(\begin{array}{c}
N \\
r+1
\end{array}\right)-1}\left|P^{j, 0}\right|^{2}\right),
$$


so

$$
\left(1+|z|^{2}\right)^{N_{0}}=\left(\sum_{j=0}^{\left(\begin{array}{c}
N \\
r
\end{array}\right)-1}\left|P^{0, j}\right|^{2}\right)\left(\sum_{j=0}^{\left(\begin{array}{c}
N \\
r+1
\end{array}\right)-1}\left|P^{j, 0}\right|^{2}\right) .
$$

Since functions on both sides of (3.12) are polynomials in variables $z$ and $\bar{z}$, where $1+|z|^{2}$ is an irreducible polynomial, one concludes

$$
\sum_{j=0}^{\left(\begin{array}{l}
N \\
r
\end{array}\right)-1}\left|P^{0, j}\right|^{2}=C_{1}\left(1+|z|^{2}\right)^{N_{1}},
$$

and

$$
\sum_{j=0}^{\left(\begin{array}{c}
N \\
r+1
\end{array}\right)-1}\left|P^{j, 0}\right|^{2}=C_{2}\left(1+|z|^{2}\right)^{N_{2}},
$$

where $C_{1}$ and $C_{2}$ are positive constants and $N_{1}, N_{2}$ are nonnegative integers with $N_{1}+N_{2}=N_{0}$. Therefore,

$$
\left\|\widetilde{F}_{r-1}\right\|^{2}=C_{1}|h|^{2}\left(1+|z|^{2}\right)^{N_{1}},
$$

and

$$
\left\|\widetilde{F}_{r}\right\|^{2}=C_{2}\left(1+|z|^{2}\right)^{N_{2}} .
$$

Now compute the metrics in terms of local coordinates. By (3.8), (3.9), (3.15) and (3.16) one gets

$$
\begin{aligned}
& F_{r-1}^{*}\left(d s_{C P}^{2}\left(\begin{array}{l}
N \\
r
\end{array}\right)-1\right)=\frac{\partial^{2} \log \left(\left\|\tilde{F}_{r-1}\right\|^{2}\right)}{\partial z \partial \bar{z}}|d z|^{2}=\frac{N_{1}}{\left(1+|z|^{2}\right)^{2}}|d z|^{2}, \\
& F_{r}^{*}\left(d s_{C P}^{2}\left(\begin{array}{c}
N+1)-1 \\
r+1
\end{array}\right)=\frac{\partial^{2} \log \left(\left\|\widetilde{F}_{r}\right\|^{2}\right)}{\partial z \partial \bar{z}}|d z|^{2}=\frac{N_{2}}{\left(1+|z|^{2}\right)^{2}}|d z|^{2},\right.
\end{aligned}
$$

and

$$
\begin{aligned}
\eta_{r-1, r}^{*}\left(d s_{C P N(r)}^{2}\right) & =\frac{\partial^{2} \log \left(\left\|\tilde{\eta}_{r-1, r}\right\|^{2}\right)}{\partial z \partial \bar{z}}|d z|^{2} \\
& =\frac{N_{0}}{\left(1+|z|^{2}\right)}|d z|^{2}=\theta \bar{\theta}
\end{aligned}
$$

Therefore

$$
F_{r-1}^{*}\left(d s_{C P}^{2}\left(\begin{array}{l}
N \\
r
\end{array}\right)-1\right)=\frac{N_{1}}{N_{0}} \theta \bar{\theta}
$$

and

$$
F_{r}^{*}\left(d s_{C P}^{2}\left(\begin{array}{c}
N \\
r+1
\end{array}\right)-1\right)=\frac{N_{2}}{N_{0}} \theta \bar{\theta}
$$


Comparing (3.20) and (3.21) with (1.15), one has

$$
q_{r-1}=\sqrt{N_{1} / N_{0}}
$$

and

$$
q_{r}=\sqrt{N_{2} / N_{0}} .
$$

By (1.14) all $q_{j}$ are locally constant. Since $q_{j}$ are continuous, they are globally constant, and $K=4 /(2 r(n-r)+n)$. In particular, $q_{0}$ is constant. But then

$$
f^{*} d s_{C P^{n}}^{2}=q_{0}^{2} f_{r}^{*} d s_{C P^{n}}^{2}
$$

and with this metric $f$ is a holomorphic curve with constant curvature $K=4 / n$ on $M$. The Theorem of Calabi now infers that $f$ is unitarily equivalent to a portion of the Veronese curve $V_{n}$ in $C P^{n}$. Pushing $f$ down to $f_{r}$ the result follows.

As an immediate corollary we recover Calabi's theorem on the rigidy of minimal 2-spheres of constant curvature in $n$-spheres.

Corollary 2. The only minimal 2-spheres of constant curvature in a standard $n$-sphere are (real) Veronese curves, up to orthogonal equivalence.

Proof. Embed the $n$-sphere into $C P^{n}$ in the standard way, and notice that the minimal 2-spheres are now pseudo-holomorphic in $C P^{n}$. By Theorem 2 they are generated by the Veronese curves in $C P^{n}$. The rest of the proof proceeds as in [1].

\section{Holomorphic CURVES IN $G(2,4)$ With CONSTANT CURVATURe 2}

Holomorphic curves in $G(2,4)$ have been studied by P. Griffiths [10] and by G. Jensen [12] in details. In this final section, we are going to follow Jensen's work to classify all holomorphic maps from $S^{2}$ to $G(2,4)$ with constant curvature 2, thus showing that Theorem 2 is no longer true for a general $G(m, N)$. In the following computation we will repeatedly use Maurer-Cartan structure equations without mentioning; details can be found in [12].

Let $f$ be a holomorphic immersion from $S^{2}$ into $G(2,4)$ and let $e$ be a local unitary frame along $f$ such that (1.7) becomes

$$
\phi=\left[\begin{array}{cccc}
\varphi_{11} & \varphi_{12} & -t_{1} \bar{\theta} & 0 \\
\varphi_{21} & \varphi_{22} & 0 & -t_{2} \bar{\theta} \\
t_{1} \theta & 0 & \varphi_{33} & \varphi_{34} \\
0 & t_{2} \theta & \varphi_{43} & \varphi_{44}
\end{array}\right]
$$

where $t_{1} \geq t_{2} \geq 0$, and $\theta$ is the unitary coframe with respect to the metric $f^{*} d s_{G(2,4)}^{2}$.

If rank $\partial f=2$, then $t_{2} \neq 0$ and $f$ is a pseudo-holomorphic curve of positive zero in $G(2,4)$. Since Gaussian curvature $K=2, t_{1} t_{2}$ is constant by 
[15]. On the other hand $f^{*} d s_{G(2,4)}^{2}=\theta \bar{\theta}=\left(t_{1}^{2}+t_{2}^{2}\right) \theta \bar{\theta}$, then $t_{1}^{2}+t_{2}^{2}=1$. Thus $0<t_{2} \leq t_{1}<1$ are constant.

If $t_{1}=t_{2}$, by [12] $f$ is locally, hence by a standard monodromy argument, globally equivalent to the curve $h: S^{2}=C P^{1} \rightarrow G(2,4)$ defined by

$$
h\left[\begin{array}{c}
z \\
w
\end{array}\right]=\left[\begin{array}{cc}
z & I_{2} \\
w & I_{2}
\end{array}\right]
$$

where $z, w \in C$ and $I_{2}$ is the $2 \times 2$ identity matrix.

Now assume that $t_{1}<t_{2}$, one can take exterior derivatives on both sides of equations $\varphi_{41}=0$ and $\varphi_{32}=0$ to get

$$
\left(t_{2} \varphi_{21}-t_{1} \varphi_{43}\right) \wedge \theta=0
$$

and

$$
\left(t_{1} \varphi_{12}-t_{2} \varphi_{34}\right) \wedge \theta=0
$$

then

$$
t_{2} \varphi_{21}-t_{1} \varphi_{43}=y \theta
$$

and

$$
t_{1} \varphi_{12}-t_{2} \varphi_{34}=z \theta
$$

where $y$ and $z$ are locally defined smooth functions, while $|y|$ and $|z|$ are globally defined on $S^{2}$.

Taking exterior derivatives on both sides of equations $\varphi_{31}=t_{1} \theta$ and $\varphi_{42}=$ $t_{2} \theta$, one obtains

$$
d\left(t_{1} \theta\right)=t_{1}\left(\varphi_{11}-\varphi_{33}\right) \wedge \theta
$$

and

$$
d\left(t_{2} \theta\right)=t_{2}\left(\varphi_{22}-\varphi_{44}\right) \wedge \theta .
$$

Using (1.4), (4.7) and (4.8) and observing that $i \omega, \varphi_{11}, \varphi_{22}, \varphi_{33}$ and $\varphi_{44}$ are purely imaginary forms, one has

$$
i \omega=\varphi_{11}-\varphi_{33}=\varphi_{22}-\varphi_{44} .
$$

It then follows from taking exterior derivatives on both sides of (4.5) and (4.6) that

$$
\left[d y+y\left(i \omega-\varphi_{11}+\varphi_{22}\right)\right] \wedge \theta=0
$$

and

$$
\left[d z+z\left(i \omega+\varphi_{11}-\varphi_{22}\right)\right] \wedge \theta=0 .
$$

By a lemma in [5], $|y|$ and $|z|$ are of analytic type. In particular, either they are identically zero or they only vanish at finitely many points. If $y z$ were not 
identically zero, then via a formula in [8], around the points where $y z$ did not vanish, one would get from (4.10), (4.11) and (1.5)

$$
\Delta \log |y z|=2 K=4>0 \text {. }
$$

Using the maximal principle agument as in [15], one then could conclude that $|y z|$ must be constant, which would lead to a contradiction since then $\Delta \log |y z|=0$. Hence $y z$ is identically zero.

If $z \equiv 0$, then taking exterior derivatives on both sides of (4.9) and using (4.5), (4.6) and (1.5), one has $|y| \equiv t_{1}^{2}-t_{2}^{2}$. By a suitable choice of a local unitary frame, one can take $y=t_{1}^{2}-t_{2}^{2}$.

Thus by [12], $f$ is unitarily equivalent to the curve $h: C P^{1} \rightarrow G(2,4)$ defined by

$$
h\left[\begin{array}{c}
z \\
w
\end{array}\right]=\left[\begin{array}{cc}
z^{2} & z \bar{w}(\cos \psi-\sin \psi) \\
-z w(\cos \psi-\sin \psi) & |z|^{2}+|w|^{2} \sin 2 \psi \\
-z w(\cos \psi+\sin \psi) & -|w|^{2} \cos 2 \psi \\
w^{2} & -\bar{z} w(\cos \psi-\sin \psi)
\end{array}\right]
$$

where $\psi$ is defined by

$$
\tan \psi=\frac{t_{1}-t_{2}}{t_{1}+t_{2}}, \quad 1>t_{1}>t_{2}>0 .
$$

The same argument applies to the case when $y \equiv 0$. In that case, $f$ is unitarily equivalent to the curve $h: C P^{1} \rightarrow G(2,4)$ defined by

$$
h\left[\begin{array}{c}
z \\
w
\end{array}\right]=\left[\begin{array}{cc}
|z|^{2}-|w|^{2} \sin 2 \Psi & z w(\cos \Psi+\sin \Psi) \\
-z \bar{w}(\cos \Psi+\sin \Psi) & z^{2} \\
\bar{z} w(\cos \Psi+\sin \Psi) & w^{2} \\
-|w|^{2} \cos 2 \Psi & z w(\cos \Psi-\sin \Psi)
\end{array}\right]
$$

where $\Psi$ is defined in (4.14). Thus, we cover the case when $t_{2} \neq 0$, i.e., all pseudo-holomorphic curves of position zero in $G(2,4)$ with curvature 2 .

Now assume that $t_{2}=0$, then $t_{1}=1$ and rank $\partial f=1$. Taking exterior derivatives on both sides of equations $\varphi_{41}=0, \varphi_{32}=0$ and $\varphi_{31}=\theta$, one gets

$$
\begin{gathered}
i \omega=\varphi_{11}-\varphi_{33}, \\
\varphi_{43}=z \theta
\end{gathered}
$$

and

$$
\varphi_{12}=y \theta
$$

where again $|z|$ and $|y|$ are well defined and are functions of analytic type on $S^{2}$.

Take exterior derivative of (4.16), then

$$
|y|^{2}+|z|^{2}=1 \text {. }
$$


If $y \neq 0$, by taking exterior derivative of (4.18), the same argument as above shows that

$$
\Delta \log |y|=4\left(1-|y|^{2}\right) \geq 0
$$

except for finitely many points on $S^{2}$. Then again $|y|$ is constant, and therefore a suitable choice of the unitary frame $e$ makes $y \equiv 1$. So by [12] $f$ is unitarily equivalent to $h: C P^{1} \rightarrow G(2,4)$ defined by

$$
h\left[\begin{array}{c}
z \\
w
\end{array}\right]=\left[\begin{array}{cc}
|z|^{2}-|w|^{2} & \sqrt{2} z w \\
-\sqrt{2} z \bar{w} & z^{2} \\
\sqrt{2} \bar{z} w & w^{2} \\
0 & 0
\end{array}\right] .
$$

If however $y=0$ and $z=1$, then $f$ is unitarily equivalent to $h: C P^{1} \rightarrow$ $G(2,4)$ defined by

$$
h\left[\begin{array}{c}
z \\
w
\end{array}\right]=\left[\begin{array}{cc}
z^{2} & 0 \\
0 & 1 \\
\sqrt{2} z w & 0 \\
w^{2} & 0
\end{array}\right] .
$$

In (4.13) if $t_{1}=t_{2}$, one gets a curve unitarily congruent to the one defined by (4.2), whereas if $t_{2}=0$ it gives a curve unitarily congruent to the one defined by (4.22). Likewise in (4.15) if $t_{2}=0$, one obtains a curve unitarily congruent to that defined by (4.21). Therefore all these curves can be uniformly written as in (4.13) and (4.15) by letting $0 \leq \psi \leq \pi / 4$ in (4.13) and $0 \leq \psi \leq \pi / 4$ in (4.15). Since each of them has invariants different from those of the others, they are not unitarily equivalent. In summary, one has

Theorem 3. There are two families of holomorphic curves with constant curvature 2 from $S^{2}$ into $G(2,4)$, which are not unitarily equivalent, and any holomorphic curve of constant curvature 2 from $S^{2}$ into $G(2,4)$ must be unitarily equivalent to exactly one of them.

\section{REFERENCES}

1. J. Bolton, G. R. Jensen, M. Rigoli and L. M. Woodward, On conformal minimal immersions of $S^{2}$ into $C P^{n}$, Math. Ann. 279 (1988), 599-620.

2. R. Bryant, Minimal surfaces of constant curvature in $S^{n}$, Trans. Amer. Math. Soc. 290 (1985), 259-271.

3. E. Calabi, Isometric embedding of complex manifolds, Ann. of Math. 58 (1953), 1-23.

4. __, Quelques applications de l'analyse aux surfaces d'aire minima, Topics in Complex Manifolds, Presses de l'Université de Montréal, pp. 58-81.

5. S. S. Chern, On the minimal immersions of the two sphere in a space of constant curvature, Problems in Analysis, Princeton Univ. Press, Princeton, N. J., 1970, pp. 27-42.

6. S. S. Chern and J. G. Wolfson, Harmonic maps of the two-sphere into a complex Grassmann manifolds. II, Ann. of Math. 125 (1987), 301-335.

7. J. Eells and J. C. Wood, Harmonic maps from surfaces to complex projective spaces, Adv. in Math. 49 (1983), 217-263. 
8. J. H. Eschenberg, I. V. Gaudalupe and R. Tribuzy, The fundamental equation of minimal surfaces in $C P^{2}$, Math. Ann. 270 (1985), 571-598.

9. M. L. Green, Metric rigidity of holomorphic maps to Kaehler manifolds, J. Differential Geom. 13 (1978), 279-286.

10. P. Griffiths, On Cartan's method of Lie groups and moving frames as applied to uniqueness and existence questions in differential geometry, Duke Math. J. 41 (1974), 775-814.

11. P. Griffiths and J. Harris, Principles of algebraic geometry, Wiley, New York, 1978.

12. G. R. Jensen, Higher order contact of submanifolds of homogeneous spaces, Lecture Notes in Math., vol. 610, Springer-Verlag, Berlin, 1977.

13. K. Kenmotsu, On minimal immersions of $R^{2}$ into $S^{n}$, J. Math. Soc. Japan 28 (1976), 182191.

14. B. Lawson, Jr., Lectures on minimal submanifolds, Vol. 1, Publish or Perish, Berkey, Calif., 1980.

15. Y. Zheng, A quantization result of curvature for holomorphic curves in Grassmann manifolds, preprint.

16. __ Harmonic maps into Grassmann manifolds, Thesis, Washington Univ., 1987.

Department of Mathematics, Washington University, St. Louis, Missouri 63130 (Current address of Quo-Sin Chi)

Current address (Yunbo Zheng): 700 W. Emerson Avenue, \#16, Monterey Park, California 91754 